\title{
Bringing Molecular Biology to Bear on Adhesion Prevention: Postsurgical Adhesion Reduction Using Intraperitoneal Inoculation of Hyaluronic Acid-Inducing Adenoviral Vector in a Murine Model
}

\author{
ROBERT A. HAMMER, M.D., ${ }^{1}$ ABRAHAM N. MORSE, M.D., ${ }^{2}$ \\ JEFFREY L. CORNELLA, M.D., ${ }^{3}$ REBECCA S. KELLER, Ph.D., ${ }^{4}$ JOSEPH HENTZ, ${ }^{5}$ \\ JOHN A. MCDONALD, M.D., Ph.D., ${ }^{6}$ and JOSEPH C. LOFTUS, Ph.D. ${ }^{7}$
}

\begin{abstract}
Objective: Seprafilm, ${ }^{\circledR}$ (Genzyme, Cambridge, MA) an absorbable adhesion barrier incorporating hyaluronic acid (HA), a high molecular mass glycosaminoglycan and important component of the extracellular matrix, has been shown to prevent adhesions in both experimental models and human subjects. Yet, the application of HA as a sheet at the time of surgery has several important logistic limitations. Recently, our laboratory has identified and cloned the genes encoding murine hyaluronic acid synthase 2 (mHAS2) and 3 (mHAS3) and engineered adenoviruses incorporating these genes, which, on intraperitoneal injection, significantly increases $\mathrm{HA}$ in peritoneal fluid. We hypothesized that intraperitoneal gene therapy with mHAS2 or mHAS3 via an adenoviral vector prior to a standardized cecal abrasion surgery would lead to a reduction in postoperative adhesion severity. Methods: Mice were assigned to one of four groups: (1) intraperitoneal inoculation with adenovirus encoding mHAS2; (2) mHAS3; (3) a control reporter adenovirus (RV) encoding GFP; or (4) intraoperative placement of a commercially available and murine-validated hyaluronic acid adhesion barrier (Seprafilm, ${ }^{\circledR} \mathrm{SF}$ ). An a priori sample size calculation was performed. Mice in groups 1, 2, and 3 underwent injection of $2 \times 10^{7}$ viral particles in $1 \mathrm{ml}$ of fluid on day -1 . Sham injection was performed on group $4 \mathrm{SF}$ mice. On day 0 , laparotomy was performed in random sequence by surgeon blinded to the experimental group. On day 7 , adhesion scores (0-3) were assigned independently by two blinded investigators. Results: Mean adhesion scores $(n=247)$ were 0.68 (mHAS2), 0.91 (mHAS3), $1.28(\mathrm{RV})$, and 0.47 (SF). Pairwise comparisons using Wilcoxon rank-sum test revealed significant reduction in severity of adhesions between mHAS2, mHAS3, and SF compared to RV $(p=0.0004,0.039$, and 0.0001 ,
\end{abstract}

\footnotetext{
${ }^{1}$ Section of Gynecology and Gynecologic Surgery, Department of Obstetrics and Gynecology, Northwestern University Medical School, Chicago, IL.

${ }^{2}$ Department of Obstetrics and Gynecology, University of Massachusetts Medical Center, Worcester, MA.

${ }^{3}$ Department of Obstetrics and Gynecology, Mayo Clinic Scottsdale, Scottsdale, AZ.

${ }^{4}$ Cardiovascular Sciences, Albany Medical College ME114, Albany, NY.

${ }^{5}$ Biostatistics, Mayo Clinic Scottsdale, Scottsdale, AZ.

${ }^{6}$ Department of Internal Medicine, University of Utah School of Medicine, Salt Lake City, UT.

${ }^{7}$ Department of Biochemistry and Molecular Biology, Mayo Clinic Scottsdale, Scottsdale, AZ.
} 
respectively). Significance persisted despite correction for multiple comparisons $(p=\mathbf{0 . 0 0 0 2}$, Kruskal-Wallis). There was a direct relationship between intraperitoneal HA concentration and adhesion reduction. Only one death (RV) was secondary to adhesive disease; differential risk of death between groups was statistically significant $(p=0.008)$ (highest in mHAS2 group). Conclusions: In a dose-response relationship, an intraperitoneal gene therapy approach to adhesion prevention in a murine model was successful, with adenoviruses most productive of $\mathrm{HA}$ resulting in the most significant reduction in adhesion scores compared to "empty" virus (RV). Although $\mathrm{SF}^{\circledR}$ best reduced postoperative adhesions, the adenoviral gene delivery approach may prove to be more effective in clinical use when peritoneal injury is less localized or at laparoscopy where the application of SF is not possible. Further studies to elucidate the reason for the differential death rates (time bias may have played a role) and to validate results are in progress. (J GYNECOL SURG 22:7)

\section{INTRODUCTION}

$\mathbf{P}$ OSTOPERATIVE ADHESIONS ACCOUNT FOR SIGNIFICANT MORBIDITY and an enormous economic burden on the health care system. Adhesions significantly increase operative time and complication risk in subsequent surgeries in abdominopelvic, cardiothoracic, and orthopedic surgery specialties and the recognized potential for adhesions can influence preoperative decisions which lead to more costly and more morbid surgical approaches. Complications from intraperitoneal adhesions after abdominopelvic surgery in the female include reduced fertility, pelvic pain, and bowel obstruction. Beyond gentle tissue handling, adjuvant adhesion prevention strategies at surgery have not consistently proven effective. Hyaluronic acid (HA), a high molecular mass glycosaminoglycan and important component of the extracellular matrix, has a slimy consistency in its hydrated state, and has been shown to prevent adhesion formation in both experimental models and human subjects. Yet, the application of HA as a sheet (Seprafilm ${ }^{\circledR}$; SF; Genzyme, Cambridge, MA) or gel (Sepracoat ${ }^{\circledR}$ and Intergel, ${ }^{\circledR}$ Genzyme) at the time of surgery has several important logistical limitations: (1) neither persists for the complete 8-10 day window when reperitonealization occurs, and (2) SF can only cover a limited portion of the injured peritoneal surface during abdominal surgery and the product, as it is currently produced, is impossible to use at laparoscopy. Yet, coverage of the entire peritoneal surface with HA is theoretically possible by manipulating the peritoneal mesothelial cells to produce HA by way of a gene therapy approach.

Previously, our laboratory has identified, sequenced, and cloned the genes encoding two of three murine hyaluronic acid synthases-hyaluronic acid synthase 2 (mHAS2) and hyaluronic synthase 3 (mHAS3) $)^{1,2}$-and constructed recombinant adenoviruses incorporating these genes. We have shown both to significantly increase HA concentration in peritoneal lavage fluid for at least 10 days following intraperitoneal injection. We have recently demonstrated a simple and reliable technique for inducing highly reproducible intraperitoneal adhesion formation in a murine model, have demonstrated the validity and interobserver consistency of an ordinal adhesion grading system, and have proven the model amenable to adhesion prevention with SF, a commercially available antiadhesion membrane currently in clinical use (hyaluronan/carboxymethylcellulose barrier $[\mathrm{HA} / \mathrm{CMC}])$, suggesting that trials of adhesion prevention technologies in this model may mirror those seen in human subjects.

We hypothesized that gene therapy of peritoneal mesothelial cells with mHAS2 or mHAS3 via intraperitoneal inoculation of adenoviral vector prior to a standardized cecal abrasion surgery would lead to a reduction in adhesion severity. This manuscript discusses our experience with a novel gene therapy approach to the prevention of postoperative adhesions. 


\section{METHODS}

\section{Candidate genes}

Murine hyaluronic acid synthase 2 [mHAS2] and 3 [mHAS3] are two of three murine isoforms of a membrane-associated protein with multiple transmembrane domains which assemble and excrete HA into the extracellular space. ${ }^{1,2}$ Sequence comparisons indicated that mHAS3 is most closely related to mHAS2 (71\% amino acid identity) and also related to mHAS1 (57\% identity). The open reading frame for mHAS3 is 1662 base pairs long and encodes a polypeptide of 554 amino acids with a predicted molecular mass of $63.3 \mathrm{kDa}$. This polypeptide is only 2 amino acids longer than the mHAS2 polypeptide. Analysis of the size distributions of hyaluronic acid generated in vitro by the recombinant proteins demonstrates that mHAS3 synthesizes hyaluronic acid with a molecular mass of $1 \times 10^{5}$ to $1 \times 10^{6} \mathrm{Da}$, shorter than those synthesized by HAS1 and HAS2 which have molecular masses of $2 \times 10^{5}$ to $\sim 2 \times 10^{6} \mathrm{Da}$. Furthermore, comparisons of hyaluronic acid secreted into the culture media by stable mHAS transfectants show that mHAS1 and mHAS3 generated hyaluronic acid with broad size distributions (molecular masses of $2 \times$ $10^{5}$ to $\sim 2 \times 10^{6} \mathrm{Da}$ ), whereas mHAS2 generates hyaluronic acid with a broad but extremely large size (average molecular mass of $>2 \times 10^{6} \mathrm{Da}$ ). ${ }^{3}$ Expression of any one of the mammalian HAS proteins in transfected mammalian cells leads to a dramatic increase in HA biosynthesis. ${ }^{2}$

\section{Creation of recombinant adenoviruses}

Replication-defective recombinant adenoviruses encoding mHAS2 or mHAS3 were generated with the AdEasy system (AdEasy, IntroGene, Baltimore, MD). ${ }^{4}$ The open reading frames (ORF) of mHAS2 or mHAS3 genes were cloned into the adenoviral shuttle vector, pShuttle-CMV, downstream from the immediate early CMV promoter. The resultant plasmids were linearized by digesting with restriction endonuclease Pme I, and subsequently cotransformed into Escherichia coli BJ5183 cells with the adenoviral backbone plasmid, pAdEasy-1. Recombinants were selected for kanamycin resistance, and recombination confirmed by restriction endonuclease analyses. Finally, the linearized recombinant plasmids were transfected into 293 cells for the production of infectious viral particles. Two hundred ninety three cells were harvested 7-10 days following transfection when cell lysis was apparent. Cell pellets were subjected to three freeze/thaw cycles and resulting viral supernatants were used to prepare high titer viral stocks by reinfection of 293 cells. Recombinant stocks were titered by plaque assay. Resultant recombinant adenoviruses are E1and E3-deficient leading to self-limiting infection. A recombinant adenovirus encoding the gene for green fluorescent protein (GFP) was used throughout the experiments as a control virus and to track the distribution of adenovirus after inoculation. Functionality of the recombinant mHAS2 and mHAS3 adenoviruses was confirmed using the red blood cell exclusion assay (Fig. 1). In addition, we demonstrated the presence of GFP at 8 days and its absence at 16 days in mouse peritoneum (data not shown).

Enzyme-linked immunosorbent assay to determine intraperitoneal hyaluronic acid production

Peritoneal concentration of HA was determined by competitive ELISA analysis. 


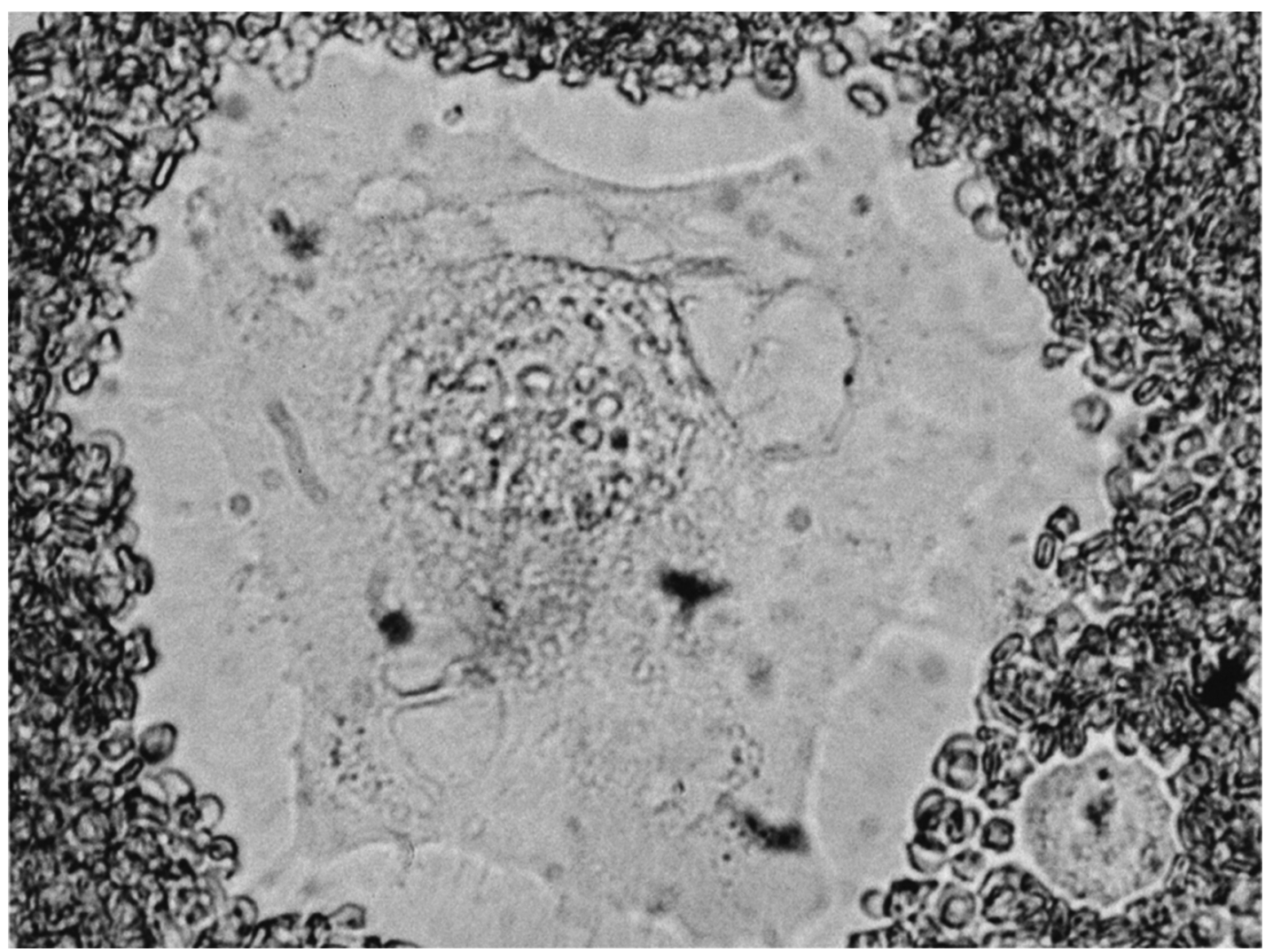

FIG. 1. Red blood cell exclusion assay. COS-1 cell infected with mHAS2 virus demonstrating extracellular HA coat in vitro.

\section{Controls}

For each experiment involving the use of mHAS-containing adenovirus, whether in ex vivo or in vivo testing, we incorporated controls using "empty" virus (same-type adenovirus without incorporated mHAS) at matched titers to demonstrate that observed effects were not due to infection by adenovirus but by expression of the encoded gene product. Preliminary experiments showed no difference between mice injected with virus-free cell lysate and mice injected with reporter virus (RV) in cell lysate, whether they had undergone surgery or not.

Mice

After weaning, FVB mice were separated by gender. Pregnant, sick, or injured mice were not used for the experiment. Mice from one cage were never mixed with mice from other cages. All mice were weighed prior to injection. Weight, gender, and age were tested in multivariate analyses and were never independent contributors to adhesion outcome differences.

\section{Protocol}

Mice were assigned to one of 4 groups: (1) intraperitoneal inoculation with adenovirus encoding mHAS2; (2) mHAS3; (3) a control reporter virus encoding GFP [RV]; or (4) intraoperative 


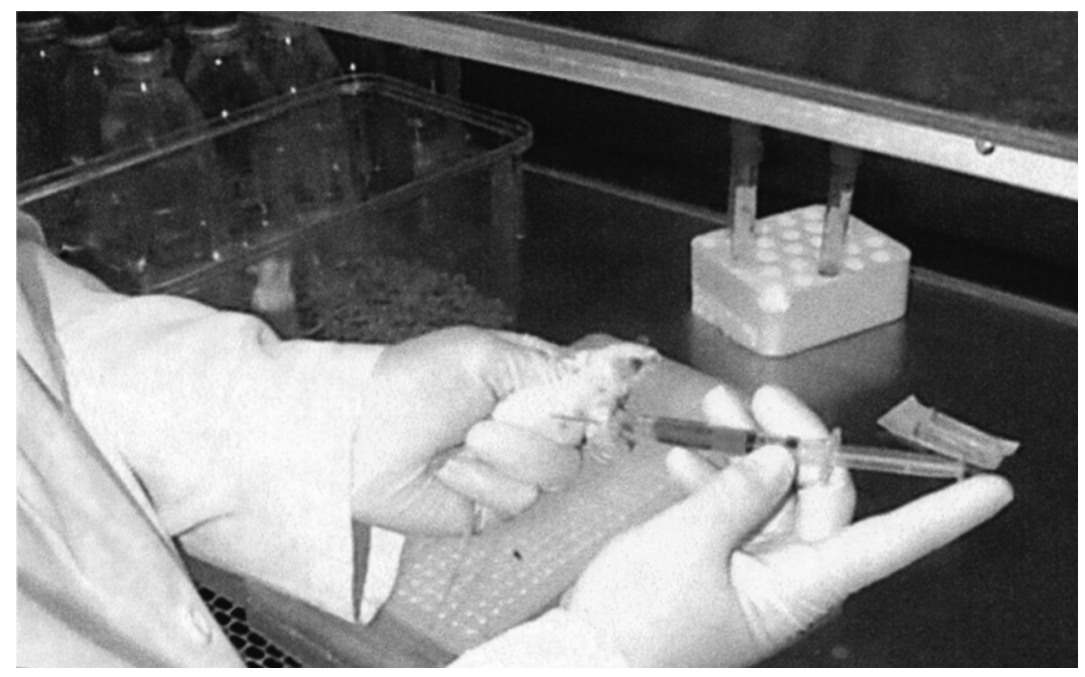

FIG. 2. Inoculation abrasion.

placement of murine-validated ${ }^{5} \mathrm{SF}$. Mice in groups 1, 2, 3 underwent injection of $2 \times 10^{7}$ viral particles in $1 \mathrm{~mL}$ of fluid on day -1 (Fig. 2). Sham injection was performed in SF mice. On day 0 , laparotomy and cecal/abdominal wall abrasion in random sequence (using a random number table) was performed by surgeon blinded to group (Figs. 3 and 4). On day 7, adhesion scores (0-3) were assigned independently by 2 blinded investigators. Viral titer of $2 \times 10^{7}$ particles $/ \mathrm{mL}$ was chosen after preliminary experiments demonstrated maximum HA production at 7 days without evidence of inflammation or death.

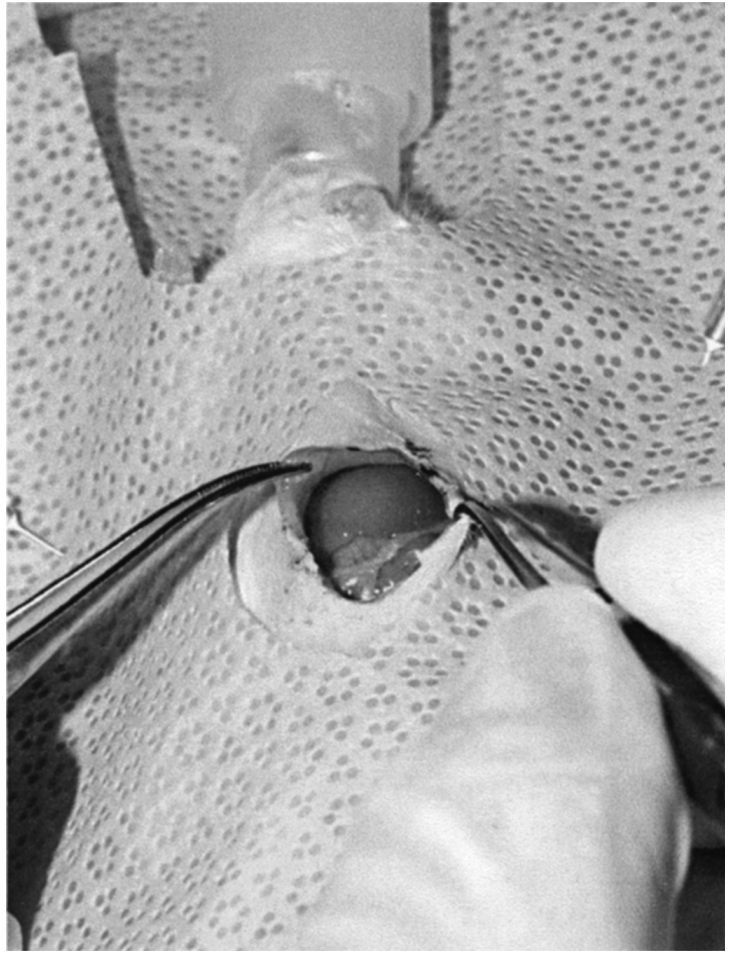

FIG. 3. Laparotomy.

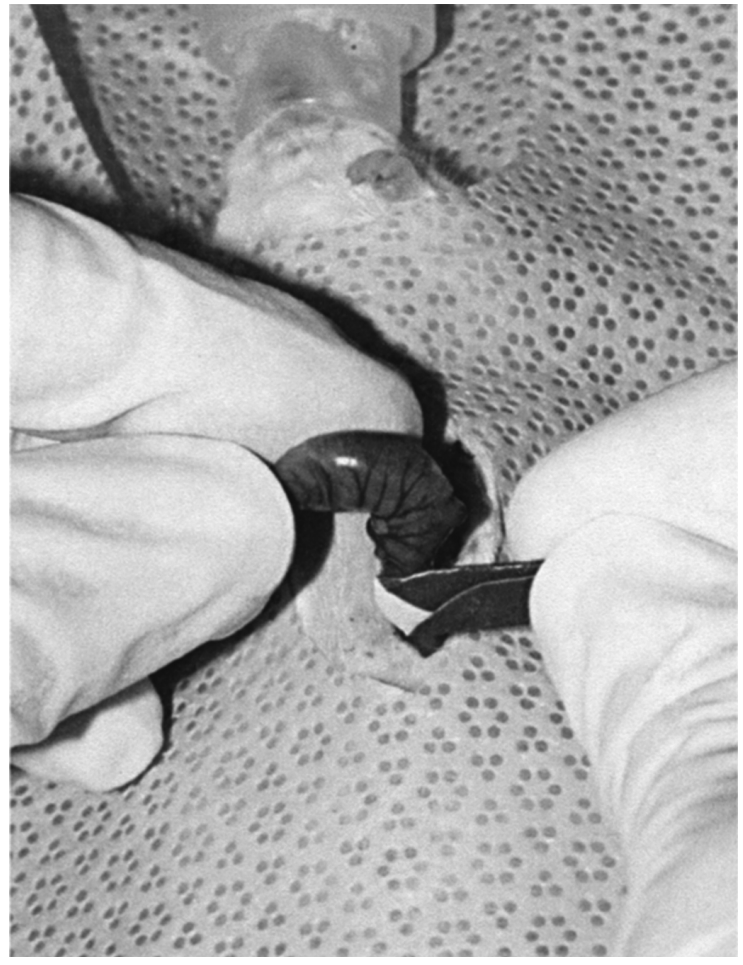

FIG. 4. Cecal. 
Surgical technique, group assignment, and blinding

All animal protocols were approved by the Mayo Clinic Institutional Animal Care and Use Committee (IACUC). We have previously reported our surgical technique for adhesion creation in the murine model..$^{5}$ Briefly, we performed laparotomy on adult male and female FVB mice in a laminar flow hood with sterile gloves, masks, and gowns. Instruments were cleaned and beadsterilized at $150^{\circ} \mathrm{C}$ between animals. Anesthesia was administered by placing each animal sequentially in a chamber with circulating $2 \%-3 \%$ isofluorane in oxygen; anesthesia was maintained with the same mixture. The skin was chemically depilated (Nair, ${ }^{\circledR}$ Carter-Wallace, Inc. Cranbury, $\mathrm{NJ}$ ), cleansed with povidone/iodine, and swabbed with $70 \%$ ethanol. The mouse was sterilely draped and a 2-cm midline laparotomy incision was made, angled toward the left upper quadrant (Fig. 3). The parietal peritoneum overlying the intra-abdominal location of the cecum (LUQ) was abraded (6 passes) using a folded 2-cm diameter circular sheet of autoclaved 400-grit aluminum oxide sandpaper. The cecum was then exteriorized and both anterior and posterior surfaces abraded (6 passes each side) to deserosalize the organ. In phase 1 of our experimentation, we demonstrated this technique to reproducibly create postsurgical adhesions $75 \%-88 \%$ of the time (mean adhesion score 2.25) with a low rate of bowel perforation and subsequent death in a murine model. ${ }^{5}$ The model was shown to be amenable to adhesion prevention with SF. We found a $49 \%$ reduction (relative risk $(\mathrm{RR})=0.51,95 \%$ confidence internal $(\mathrm{CI})=0.30$ to 0.87 ) in adhesion formation with the application of SF after cecal abrasion surgery, similar to reported rates in several clinical trials of SF. ${ }^{6}$ Only after abrasion was completed was allocation to SF group (or not) revealed. If assigned to SF group, a $2-\mathrm{cm} \times 2-\mathrm{cm}$ sterile piece of SF was applied around the cecum and moistened with three drops of saline. The cecum of non-SF animals were moistened with three drops of saline. The cecum was replaced and the abdominal incision closed in two layers using a running 5-0 Vicryl (polyglactin 910, Ethicon, Somerville, NJ) for the fascia and skin. Anesthesia induction, preparation, laparotomy, abrasion, and closure required approximately 6-7 minutes per animal. Animals received buprenorphine $0.25 \mathrm{mg} / \mathrm{kg}$ immediately prior to incision and acetaminophen $1 \mathrm{mg} / \mathrm{mL}$ in their drinking water for the first 3 postoperative days. On day 7 , mice were euthanized by carbon dioxide narcosis and adhesion scores were assigned separately by two trained investigators blinded to treatment group and the other investigator's scores. Differences were resolved by assigning the mean adhesion score to that subject. No scores for the same animal differed by more than one grade. As previously reported, ${ }^{5}$ we used a 4-tiered adhesion scoring system for grading the severity of adhesions involving the cecum based on that published by Montz and Gotlieb. ${ }^{7}$ The scoring system was defined a priori as follows:

- Grade 0-No adhesions

- Grade 1-filmy adhesions, easily separated with gentle traction

- Grade 2-moderate adhesions, requiring significant traction to separate

- Grade 3-dense adhesions, unable to separate without tearing underlying involved structures.

\section{Minimization of bias}

All efforts were made to minimize bias. Each injection/surgery/sacrifice "session" always included mice from the RV group, the SF group, and at least one of the mHAS groups. After injection of mice, cage cards identifying group assignment were removed by personnel not involved in the surgery or adhesion scoring, and cages were rearranged and recodified. Group assignment could not be surmised by color of cage, size, age, gender of mice, or number of mice per cage. Surgery was performed by one surgeon in an order determined by random number table. At the time of sacrifice and scoring, the investigators were blinded to each animal's group assignment. 
Deaths and histologic evaluation

Cages were checked daily. In the event of death, cage, date, and external characteristics were recorded. The laparotomy incision was reopened and an attempt was made to determine cause of death; dilated bowel loops proximal to an adhesion, torsion, or narrowing were considered evidence of death due to adhesions. Evidence of stool in the abdomen and cecal perforation on one of the first two days after surgery suggested bowel injury from cecal abrasion as the cause of death. Histologic sections of liver, diaphragm, heart, lung, and brain were examined for evidence of infection. In a few cases, transient dissemination of reporter virus to liver and spleen without evidence of inflammatory response was seen. There were no other histologic findings.

\section{Statistical analysis}

An a priori sample size calculation was performed: Thirty mice were needed in each group to have $80 \%$ power to detect a $40 \%$ reduction in the rate of any adhesion formation $\left(P_{0}=0.9 ; P_{1}=0.5\right)$ with an $\alpha$ of 0.05 . Because previous experiments demonstrated a greater difference between control and SF mice, only 15 mice were needed in the SF group to detect the same difference with the same alpha and beta. To maintain the presence of mHAS, RV, and SF groups in all sessions to assure blinding, total number per group varied but surpassed that required by the power calculation.

The difference in adhesion grade between groups was compared using the Wilcoxon rank-sum test. The Chi-square and Fisher's exact tests was employed to compare the incidence of adhesions in the two groups. Death rates were compared using Chi-square test for homogeneity of distributions.

\section{RESULTS}

\section{Demonstration of productive infection HA in vivo assays}

Mice $(n=90)$ were randomly assigned to receive intraperitoneal injection of one of the inoculation solutions. Control injections were also tested, including sham injections and injection with cell lysate with virus and with RV plus cell lysate. Mice were sacrificed at 3; 7; and 10-day intervals. Peritoneal lavage was performed using $3 \mathrm{ml}$ of sterile saline. Peritoneal concentration of HA was determined by competitive ELISA analysis. Results are presented in Table 1. Significantly higher peritoneal HA concentration was noted at all intervals after mHAS2 inoculation compared to all other groups (Student's $t$-test). For comparison, a prior experiment of ten mice per group to determine mean HA concentrations of peritoneal lavage fluid in mice undergoing cecal/abdominal wall abrasion surgery with and without placement of SF and control mice not having surgery demonstrated the following at 3 days: with surgery/SF, mean HA concentration $2421 \pm$ $1035 \mathrm{ng} / \mathrm{ml}$; with surgery/no SF, mean HA concentration $1416 \pm 1302 \mathrm{ng} / \mathrm{mL}$; control mice (no surgery), mean HA concentration $447 \pm 74$.

Demonstration of adhesion prevention with adenoviral vectors

Age of mice ranged from 70 to 120 days (mean $88.5 \pm 14.7$ days). Weight ranged from 16 to $32 \mathrm{~g}$ (mean $23.9 \pm 3.6 \mathrm{~g}$ ). Gender was evenly male $(50 \%)$ and female $(50 \%)$ overall. There 
were no statistical differences in age, weight, and gender between groups. Adhesion score data is presented in Tables 2 and 3. Two hundred and forty-seven (247) mice were in the experiment. Deaths were observed in $22(30.6 \%)$ in mHAS2 group, $16(25.4 \%)$ in mHAS3 group, 5 $(8.5 \%)$ in RV group, and 10 (18.9\%) in SF group. In only one mouse was the suspected cause of death adhesion-related. One hundred ninety four mice were evaluable on day 7 for adhesion scoring. In group 1 (mHAS2), the number of evaluable mice $(n)$ was 50 , mean adhesion score was 0.68 , and $28 \%$ of mice had adhesion scores greater than grade 1 . The values for remaining groups were as follows: group 2 (mHAS3), 47, 0.91, and 40\%; group 3 (RV), 54, 1.28 , and $48 \%$; and group 4 (SF), 43, 0.47, and 12\%. Pairwise comparisons with RV (Table 3 ) demonstrated a statistically significant reduction in adhesion severity in mHAS2 group $(p<$ $0.001)$, mHAS3 group $(p=0.03)$, and SF group $(p<0.001)$. Statistical significance persisted despite correction for multiple comparisons (Kruskal-Wallis, $p=0.002$ ) and with nonparametric (Chi-square) testing comparing rates of adhesion grade greater than 1. Statistical testing of the difference in rates of adhesion grade greater than 1 between mHAS2-2 and SF groups was $p=0.051$ (Chi-square). Adhesion prevention was inversely proportional to amount of HA production (data not shown). Differential risk of death between groups was statistically significant ( $p=0.008$, Chi-Square test for homogeneity of distributions), highest in mHAS2-2 group. However, death rates between mHAS2 and SF groups was not statistically different $(0.14$, Chisquare).

\section{DISCUSSION}

Post-surgical adhesions continue to represent a formidable challenge to surgeons, causing significant direct and indirect morbidity. Intraperitoneal adhesions are the leading cause of bowel obstruction in the developed world. ${ }^{8}$ A recent survey estimated that there are 12,000-14,000 cases of adhesive bowel obstruction annually in the United Kingdom. ${ }^{9}$ In the United States, there were more than 130,000 admissions for adhesive bowel obstruction in 1990. One percent (1\%) of all surgical admissions and 3\% of all laparotomies were secondary to complications from adhesive disease. In the United States in 1988, the direct hospital costs for the surgical treatment of abdominal adhesions exceeded 1.1 billion dollars. ${ }^{10}$ Beyond gentle tissue handling and meticulous surgical technique, adjuvant interventions are essential to reduce this morbidity.

The cascade of adhesion formation begins with trauma to the peritoneal surface at surgery. Extracellular matrix and thromboplastin are exposed which leads to the activation of the clotting cascade. A fibrin gel matrix forms between injured surfaces. Via a complex interplay of plasminogen, plasminogen activator, and plasminogen activator inhibitors, ensuing fibrinolytic activity can break down the fibrin gel before an adhesion is formed. In the presence of ischemia and necrosis, however, fibrinolytic activity is impaired and the fibrin gel matrix is subsequently invaded by

Table 1. In Vivo HA Production (ng/mL)

\begin{tabular}{lrrr}
\hline Group & 3-day & 7-day & 10-day \\
\hline Cell lysate & 38 & 38 & 70 \\
RV & 73 & 208 & 65 \\
mHAS3 & 1,571 & 1,100 & 442 \\
mHAS2 & $13,360^{*}$ & $6,271^{*}$ & $3,426^{*}$ \\
\hline
\end{tabular}

$* p<0.05$ compared to all other groups at same time interval (Student's $t$-test). HA, hyaluronic acid; $\mathrm{RV}$, reporter adenovirus. 
Table 2. Adhesion Scores by Group

\begin{tabular}{lllcc}
\hline & mHAS2 & mHAS3 & $R V$ & $S F$ \\
\hline $\mathrm{N}$ & 72 & 63 & 59 & 53 \\
Mortality* & $22(31 \%)$ & $16(25 \%)$ & $5(8 \%)$ & $10(19 \%)$ \\
Evaluable (n) & 50 & 47 & 54 & 43 \\
Adhesion score; mean (SD) & $0.68(0.72)$ & $0.91(0.76)$ & $1.28(0.81)$ & $0.47(0.65)$ \\
Any adhesion & $56 \%(28 / 50)$ & $66 \%(31 / 47)$ & $87 \%(47 / 54)$ & $42 \%(18 / 43)$ \\
Adhesion grade $>1$ & $28 \%(14 / 50)$ & $40 \%(19 / 47)$ & $48 \%(26 / 54)$ & $12 \%(5 / 43)$ \\
\hline
\end{tabular}

$* p=0.008$, Chi-square

$\mathrm{SD}$, standard deviation.

fibroblasts, collagen is deposited, and capillaries form, leading to permanent adhesions. A variety of pharmacologic agents, solutions, and physical barriers have been proposed and tested for adhesion prevention; none has been shown to be consistently effective. ${ }^{11}$ Hyaluronic acid, a nontoxic and nonimmunogenic naturally occurring glycosaminoglycan ubiquitous in the extracellular matrix of all tissues, is synthesized by a transmembrane synthase enzyme which adds disaccha-

Table 3. Comparison of Adhesion Outcomes Between Groups

\begin{tabular}{|c|c|c|c|}
\hline & Difference & $\mathrm{p}^{*}$ & $95 \% C I$ \\
\hline \multicolumn{4}{|l|}{ Adhesion score; mean } \\
\hline mHAS3 vs RV & -0.37 & 0.03 & NA \\
\hline mHAS2 vs RV & -0.60 & $<0.001$ & NA \\
\hline SF vs RV & -0.81 & $<0.001$ & NA \\
\hline mHAS3 vs SF & -0.44 & 0.006 & NA \\
\hline mHAS2 vs mHAS3 & -0.23 & 0.14 & NA \\
\hline mHAS2 vs SF & -0.21 & 0.12 & NA \\
\hline \multicolumn{4}{|c|}{ Incidence any adhesion; proportion } \\
\hline mHAS3 vs RV & -0.21 & 0.01 & $-0.37-0.05$ \\
\hline mHAS2 vs RV & -0.31 & $<0.001$ & $-0.47-0.15$ \\
\hline SF vs RV & -0.45 & $<0.001$ & $-0.62-0.28$ \\
\hline mHAS3 vs SF & 0.24 & 0.02 & $0.04-0.44$ \\
\hline mHAS2 vs mHAS3 & -0.10 & 0.32 & $-0.29-0.09$ \\
\hline mHAS2 vs SF & 0.14 & 0.17 & $-0.06-0.34$ \\
\hline \multirow{2}{*}{\multicolumn{4}{|c|}{$\begin{array}{l}\text { Incidence adhesion grade }>1 \text {; proportion } \\
\text { mHAS } 3 \text { vs RV }\end{array}$}} \\
\hline & & & \\
\hline \multicolumn{4}{|c|}{ mHAS2 vs RV } \\
\hline \multicolumn{4}{|l|}{ SF vs RV } \\
\hline \multicolumn{4}{|l|}{ mHAS3 vs SF } \\
\hline \multicolumn{4}{|l|}{ mHAS2 vs mHAS3 } \\
\hline \multicolumn{4}{|l|}{ mHAS2 vs SF } \\
\hline \multicolumn{4}{|l|}{ Mortality; proportion } \\
\hline mHAS3 vs RV & 0.17 & 0.01 & $0.04-0.30$ \\
\hline mHAS2 vs RV & 0.22 & 0.002 & $0.09-0.35$ \\
\hline SF vs RV & 0.10 & 0.11 & $-0.02-0.24$ \\
\hline mHAS3 vs SF & 0.07 & 0.40 & $-0.09-0.21$ \\
\hline mHAS2 vs mHAS3 & 0.05 & 0.51 & $-0.10-0.20$ \\
\hline mHAS2 vs SF & 0.12 & 0.14 & $-0.03-0.26$ \\
\hline
\end{tabular}

* $p$-values for adhesion scores were calculated by using the Wilcoxon rank-sum statistic. $p$ values and confidence intervals for adhesion incidence and mortality were calculated using the Pearson chi-square statistic.

CI, confidence interval. 
ride units of D-glucuronic acid and D-N-acetylglucosamine to a growing HA molecule which is extruded into the extracellular matrix. As an adhesion prevention adjunctive agent, HA creates a hydrogel layer and prevents tissue-tissue apposition during the process of reperitonealization or repair of injured surfaces. Precoating tissues with HA solutions has been shown by some to reduce, but not eliminate, adhesion formation. ${ }^{9}$

$\mathrm{SF}$ is an adhesion barrier (one of two barriers currently approved by the FDA for adhesion prevention) in which HA is covalently combined with carboxymethylcellulose (CMC) and chemically modified to reduce water solubility and prolong in vivo residence. It becomes a gel within 24-48 hours, is effective in the presence of blood, and is absorbable, remaining for 7 days, being excreted through the kidneys by 28 days. ${ }^{11}$ It has been shown to be a clinically effective adhesion barrier ${ }^{12}$ in human subjects and we have confirmed its efficacy in the murine model. There are, however, significant limitations to SF and the other currently available interventions, including the inability to readminister solutions applied at the time of surgery (Sepracoat and Intergel), lack of persistence of adhesion barriers for the necessary 8-10-day window and the inability to direct to more than a fraction of the peritoneal surface (SF), inability to use in the presence of blood (Interceed ${ }^{\circledR}$ ), requirement of suturing and subsequent removal (Gore-Tex ${ }^{\circledR}$ ), and difficulty in handling, especially at laparoscopy (SF). The ideal system would provide complete peritoneal coverage, persist for at least 8-10 days following administration, and be easy to apply at laparotomy or laparoscopy.

Manipulating the peritoneal mesothelium by adenoviral transfection with a promiscuous CMV promoter upstream from a hyaluronic acid synthase gene is a novel approach to adhesion prevention. Adenoviruses are a large group of nonenveloped, regular icosahedron-shaped doublestranded DNA viruses and have shown promise as vectors in gene therapy programs because they are easy to work with, infect most mammalian cell types, can induce host cell protein synthesis, and can be made replication-deficient to prevent proliferation and assure a self-limited infection. Using reporter viruses (e.g., GFP or $\beta$-galactosidase), we documented peritoneal transfection. Histologic examination of the peritoneum demonstrated only focal inflammation and the histopathologist felt this was likely due to the physical injury from injection. Histologically, generalized peritonitis was not observed in any of the experimental animals.

In this paper, we have demonstrated in a murine model significant adhesion prevention using intraperitoneal inoculation of hyaluronic acid-inducing adenoviral vector (47\% reduction in mean adhesion score between mHAS2 and RV, $42 \%$ reduction in mice with adhesion grades of 2 or greater). Greater HA production correlated directly with more effective adhesion prevention. While greater adhesion prevention may be possible with higher and more purified titers of mHAS2 adenovirus, this will need to be tested experimentally; a plateau of effectiveness is anticipated when the inflammatory effects of inoculation (or higher titers of HA) begin to outweigh the beneficial properties of HA in preventing tissue apposition. While SF was most effective in prevention of adhesion formation, limitations of use noted above make SF an imperfect player in the struggle to prevent the morbidity related to post-surgical adhesions.

The markedly increased mortality in the mHAS2 arm of the study is of concern for two reasons. First, it may have biased our results if mice with a higher adhesion grade were more likely to die, thus artificially lowering the apparent adhesion score in the mHAS2 group. This seems unlikely since postmortem necroscopy did not show evidence of adhesion formation. Second, while we had no deaths in experiments demonstrating productive infection (HA in vivo assays), it raises questions as to whether the increased mortality might represent a real effect of inoculation with such highly productive viruses and this would have negative implications for its clinical use. The viral solution we used had 293 cellular elements; whether a highly purified viral preparation would negate this effect will need to be tested. Importantly, death rates in subsequent experiments using the same viral samples and same technique have been negligible and the newest data, to be submitted when complete, continue to confirm substantial reductions in adhesion grade in highly 
productive HA viruses. We did demonstrate GFP from the peritoneal inoculation RV in liver and spleen at 48 hours after intraperitoneal injection. While these findings need to be confirmed and the impact of such HA productive viruses, while replication-deficient, is not known, at this time, we do not believe that viremia or dissemination of large quantities of virus played a significant role in death rates. Because of the timing of availability of the viral innoculum, early experiments combined RV, SF, and mHAS3 groups, while late experiments included more of the mHAS2. For this reason, it is our suspicion that time bias may have played a significant role.

In an effort to understand its greater ability to reduce adhesions and to improve on our own viral prevention intervention, we plan to study the mechanical role of the carboxymethylcellulose membrane in the efficacy of SF (to separate its effect from the effect of HA). We have begun to investigate the effect of timing of injection in relation to surgery, but have yet to find a significant difference. We anticipate testing our methods next in a larger animal model and have begun to assess whether there are potential long-term problems with intraperitoneal adenoviral inoculation.

While we continue to perfect and test the safety and efficacy of our experimental gene therapy approach to adhesion prevention, we believe that alteration of the peritoneum in situ provides a unique locus to direct efforts at post-surgical adhesion reduction and adenoviral vectors for delivery of HA synthase genes and has the potential for wide clinical applicability for use in the reduction of postsurgical adhesions in abdominal, gynecologic, cardiac and orthopedic surgeries.

\section{ACKNOWLEDGMENTS}

To A.N. Morse and J.A. MacDonald, without whose initial work, and to R.S. Keller and J.C. Loftus, without whose daily guidance and adenoviral expertise, this project would not have come to fruition.

\section{REFERENCES}

1. Spicer AH, Augustine ML, McDonald JA. Molecular cloning and characterization of a putative mouse hyaluronan synthase. J Biol Chem 1996;271:23400.

2. Spicer AP, Olson JS, McDonald JA. Molecular cloning and characterization of a cDNA encoding the third putative mammalian hyaluronan synthase. J. Biol Chem 1997;272:8957.

3. Itano N, Sawai T, Yoshida M, et al. Three isoforms of mammalian hyaluronan synthases have distinct enzymatic properties. J Biol Chem 1999;274:25085.

4. He, TC, Zhou S, da Costa LT, Kinzler KW, Vogelstein, BA. Simplified system for generating recombinant adenoviruses. Proc Natl Acad Sci USA, 1998;95:2509.

5. Morse AN, Hammer RA, Cornella JL, Loftus JC. Validation of a mouse adhesion reduction model using Seprafilm. J Gynecologic Surgery, 2005;21:147.

6. Diamond MP, and the Sepracoat Adhesion Study Group. Reduction of de novo post-surgical adhesions by intraoperative precoating with Sepracoat (HAL-C) solution: A prospective, randomized, blinded, placebo-controlled multicenter study. Fertil Steril 1998;69:1067.

7. Montz FJ, Holschneider CH, Bozuk M, Gotlieb WH, Martinez-Maza O. Interleukin 10: Ability to minimize postoperative intraperitoneal adhesion formation in a murine model. Fertility \& Sterility. 1994;61:1136.

8. Ellis H. The prevention and treatment of adhesions. Infect Surg 1983;803.

9. Homdahl L, Risberg B, Beck DF, et al. Adhesions: Pathogenesis and prevention-panel discussion and summary [review]. Eur J Surg Suppl 1997;577:56. 
10. Ray NF, Larsen JW, Stillman RJ, Jacobs RJ. Economic impact of hospitalizations for lower abdomnal adhesiolysis in the United States in 1988. Surg Gynecol Obstet 1993;176:271.

11. Burns JW, Colt MJ, Burgees LS, Skinner KC. Preclinical evaluation of Seprafilm bioresorbable membrane. Eur J Surg 1997;577(Suppl)40.

12. Becker JM, Dayton MT, Fazio VW, et al. Prevention of postoperative abdominal adhesions by a sodium hyaluronatebased bioresorbable membrane. A prospective, randomized, double-blind multicenter study.[comment]. J Am Coll Surg 1996;183:297.

Address reprint requests to:

Robert A. Hammer, M.D.

Section of Gynecology and Gynecologic Surgery Department of Obstetrics and Gynecology Northwestern University Medical School 680 North Lake Shore Drive, Suite 1015 Chicago, IL 60611

E-mail: RHammer@nmff.org 